\title{
Early medication use in new-onset rheumatoid arthritis may delay joint replacement: results of a large population-based study
}

Cristiano S. Moura ${ }^{1,2}$, Michal Abrahamowicz ${ }^{1,2}$, Marie-Eve Beauchamp², Diane Lacaille², Yishu Wang ${ }^{1}$, Gilles Boire ${ }^{4}$, Paul R. Fortin ${ }^{5}$, Louis Bessette ${ }^{5}$, Claire Bombardier ${ }^{6}$, Jessica Widdifield ${ }^{7}$, John G. Hanly ${ }^{8}$, Debbie Feldman ${ }^{9}$, Walter Maksymowych ${ }^{10}$, Christine Peschken ${ }^{11}$, Cheryl Barnabe ${ }^{12,13}$, Steve Edworthy ${ }^{12,13}$, Sasha Bernatsky ${ }^{1,2^{*}}$ and CAN-AIM

\begin{abstract}
Introduction: Use of disease-modifying anti-rheumatic drugs (DMARDs) in rheumatoid arthritis (RA) may prevent joint damage and potentially reduce joint replacement surgeries. We assessed the association between RA drug use and joint replacement in Quebec, Canada.

Methods: A cohort of new-onset RA patients was identified from Quebec's physician billing and hospitalization databases from 2002-2011. The outcome was defined using procedure codes submitted by orthopedic surgeons. Medication use was obtained from pharmacy databases. We used alternative Cox regression models with time-dependent variables measuring the cumulative effects of past use during different time windows (one model focussing on the first year after cohort entry) for methotrexate (MTX), and other DMARDs. Models were adjusted for baseline sociodemographics, co-morbidity and prior health service use, time-dependent cumulative use of other drugs (anti-tumor necrosis factor [anti-TNF] agents, other biologics, cyclooxygenase-2 inhibitors [COXIBs], nonselective nonsteroidal antiinflammatory drugs [NSAIDs], and systemic steroids), and markers of disease severity.

Results: During follow-up, 608 joint replacements occurred among 11,333 patients (median follow-up: 4.6 years). The best-fitting model relied on the cumulative early use (within the first year after cohort entry) of MTX and of other DMARDs, with an interaction between MTX and other DMARDs. In this model, greater exposure within the first year, to either MTX (adjusted hazard ratio, HR= 0.95 per 1 month, $95 \%$ confidence interval, $95 \%$ Cl 0.93-0.97) or other DMARDs ( $H R=0.97,95 \% \mathrm{Cl}$ 0.95-0.99) was associated with longer time to joint replacement.
\end{abstract}

Conclusions: Our results suggest that longer exposure to either methotrexate (MTX) or other DMARDs within the first year after RA diagnosis is associated with longer time to joint replacement surgery.

\section{Introduction}

Rheumatoid arthritis (RA) is a serious inflammatory arthritis and affects $1 \%$ of the population in the developed world [1]. RA is characterized by joint pain and swelling, which may result in physical impairments, joint deformity, disability, and decreased quality of life. The pain and loss

\footnotetext{
* Correspondence: sasha.bernatsky@mcgill.ca

'Department of Epidemiology, Biostatistics and Occupational Health, McGill University, Montreal, QC, Canada

${ }^{2}$ Division of Clinical Epidemiology, McGill University Health Centre, 687 Pine Avenue West, V-Building (V2.09), Montreal, QC H3A 1A1, Canada

Full list of author information is available at the end of the article
}

of function in RA largely correlates with the extent of uncontrolled disease activity.

Treatment strategies for RA have improved dramatically over the past decade. Furthermore, earlier treatment increases the chance of clinical response and disease remission $[2,3]$ compared to the same treatment administered later in the disease course [4]. Disease-modifying antirheumatic drugs (DMARDs) include methotrexate (MTX), which is widely considered the cornerstone in RA care.

Uncontrolled RA activity can lead to irreversible joint damage requiring joint replacement surgery [5]. There are 
increasing randomized controlled (and observational) data suggesting that early and targeted treatment decreases joint damage in RA. It is hoped that prompt initiation of therapy and better control of disease could ultimately decrease the long-term need for orthopedic surgery. Population-based studies have demonstrated that orthopedic surgery rates have declined alongside the concomitant increase in anti-rheumatic drug use [6], however, it is uncertain to what extent early therapy ultimately reduces the need for later joint replacement surgery in RA.

In this study, we hypothesized that early drug exposure soon after RA diagnosis reduces the risk of subsequent joint replacement surgery. To assess this issue, we studied the potential association between RA drug use and joint replacement using a population-based RA cohort assembled from health administrative databases in Quebec.

\section{Methods}

\section{Data sources}

We performed cohort analyses on new-onset RA patients with public prescription drug coverage identified in the Quebec Health Insurance Program (RAMQ) databases from 2002-2011. In principle, as in each province in Canada, all Quebec residents (approximately 7.6 million persons) have access to comprehensive health care in terms of physician visits and hospitalization. Administrative databases record hospitalization and physician billing data for all residents of Quebec. The data include hospitalization discharge diagnoses (a primary diagnosis and up to 15 non-primary diagnoses per hospitalization, abstracted by medical records clerks), and physician-visit billing-claim diagnostic codes (a single diagnostic code is allowed per visit). Those residents who are beneficiaries of the provincial drug plan (which includes all seniors, and any non-seniors without private drug insurance, representing approximately $40 \%$ of residents) can also be linked to the province's dispensed prescription claims database (which includes information about drug, dose, duration, and dates).

We received ethics approval from the Quebec Commission for Access to Information. All data were denominalized, and identifying health card numbers were scrambled by the Quebec Health Insurance Board. As the data are anonymous, no informed consent is required from individual patients.

\section{Study population}

To establish a population-based incident RA sample, all physician visits with an RA diagnosis code between 1 January 2002 and 31 December 2011 were identified. To increase the positive predictive value and specificity of the RA case definition, cases required at least three visit billing codes using International Classification of Diseases (ICD)-9, code 714 over a 3-year period, at least one of which was by an internist or a rheumatologist. This represents an adaptation of the RA diagnosis that was recently validated by Widdifield et al. (2013) using Ontario administrative data [7]. To identify truly incident (rather than prevalent) RA cases, we removed any patient who had any billing codes for RA prior to 1 January 2002, as data were available from 1989. Patients were followed from cohort entry (time when they fulfilled the RA case definition) until their first joint replacement surgery, or were censored at death date, or the end of study period (31 December 2011), whichever came first. We restricted our analyses to those patients who had drug coverage by the public drug program at the cohort entry and during at least $80 \%$ of their follow-up time and who had follow up longer than 1 year.

\section{Exposure assessment}

For each prescription of MTX, or other DMARDs (sulfasalazine, chloroquine, hydroxychloroquine, leflunomide, cyclosporine, minocycline, penicillamine, and cyclophosphamide), anti-TNF inhibitors, other biologic DMARDs (anakinra, rituximab, abatacept) cyclooxygenase-2 inhibitors (COXIBs), nonselective nonsteroidal anti-inflammatory drugs (NSAIDs) and systemic steroids, the start date, number of pills, dosage, and days supplied were retrieved from the prescription claims and used to construct the daily drug exposure matrix [8]. The daily exposure matrix was then used to calculate time-dependent measures of cumulative duration of use of a specific drug, or class of drugs until a given day during the follow up [9]. For overlapping prescriptions of the same drug, the individual was assumed to have had prescriptions refilled early and completed the first prescription before starting the second. The same rule was applied to overlapping prescriptions of the same drug but with different doses and overlapping prescriptions for different drugs within the same drug class (anti-TNF agents, systemic steroids, COXIBs or NSAIDs). However, given that combination use is common for DMARDs, prescriptions for each class of DMARD were treated separately. When there was a gap of 7 days or less between two prescriptions of the same drug, or of different drugs within the same drug class (excluding DMARDs), it was assumed that the drug was taken continuously and the gap was filled with the daily dose of the second prescription. Exposures to our main anti-rheumatic drugs included 1) MTX and 2) other DMARDs besides MTX.

\section{Outcomes}

The outcome of interest was the time from cohort entry to the first joint replacement surgery (for any joint), defined using the Canadian Classification of Health Intervention (CCI) and the Canadian Classification of Diagnostic, Therapeutic and Surgical Procedures (CCP) procedure codes for joint replacement (see Appendix 1). 


\section{Covariates}

Variables that were available in the administrative databases and considered potential confounders for the association between the drugs of interest and joint replacement were selected a priori and adjusted for in all the multivariable models. These included sex, age at cohort entry (in years and with a squared-age term added to account for non-linear effects), place of residence (urban or rural, defined from postal codes) social assistance status at cohort entry, and ecological measures based on census data, on income, education level, and employment rate in the area. We used diagnostic codes from all outpatient physician and/or hospital visits during the 3 years before cohort entry to assess comorbidities (including osteoarthritis (OA), myocardial infarction, diabetes, osteoporosis, cerebrovascular disease, acute renal failure, chronic renal failure, coronary artery disease, chronic obstructive pulmonary disease (COPD), asthma, cancer and the Charlson index). To discriminate between high and low users of the health care system, we adjusted for a binary indicator of high users, defined as people with at least 20 physician visits in at least 1 year of the 3 years before baseline. To adjust for disease severity, we used a time-varying variable capturing the number of rheumatologist visits during the follow-up period (log-transformed). We also controlled for a time-varying indicator of the presence of extraarticular manifestations of the disease during the follow up; this included rheumatoid lung, Felty's syndrome, rheumatoid carditis, eye involvement, dermatological complications (vasculitis, pyoderma gangrenosum), neuropathies and amyloidosis. Finally, we adjusted for timedependent variables reflecting cumulative use of other drugs, anti-TNF inhibitors, other biologics, COXIBs, NSAIDs, and systemic steroids during the follow up, and binary indicators of anti-TNF, MTX, other DMARDs, COXIBs, NSAIDs, and systemic steroid use during the period of 1 year before cohort entry.

\section{Statistical analyses}

Descriptive statistics were used to characterize the study population. We used a Cox proportional hazards $(\mathrm{PH})$ regression model with time-dependent variables measuring drug use for 1) MTX and 2) other DMARDs. As mentioned, the model also controlled for concomitant drug exposure (anti-TNF inhibitors, other biologics, COXIBs, NSAIDs, steroids). Our primary analyses considered the effects of early use of MTX and/or DMARDs, that is, in the first year of follow up only. Different time windows for cumulative drug use were considered in alternate models [10]: 1) during the second year of follow up, 2) throughout the entire follow up, and 3) throughout follow up but not including the year prior to the index time for each event. The cumulative use was obtained by summing the duration of all prescriptions for the relevant drugs, up to a given day over the relevant time period. We also tested for an interaction term between the cumulative effects of MTX and other DMARDs to account for possibly increased or decreased risks among users of both drug classes. Adjusted hazard ratios (HRs) with $95 \%$ CIs were generated. The fit to the data of the different models was compared with the Akaike Information Criterion (AIC) [11].

In sensitivity analyses, we weighted the cumulative exposure to evaluate if weighting past exposure by recent use would improve the prediction of joint replacement surgery [12]. This method estimates from the data the relative weights of the timing of past exposure of each of MTX and other DMARDs on risk of outcome. We also performed sensitivity analysis excluding subjects with previous diagnosis of OA or excluding the cases of hip replacements, as these are less likely to be attributed to RA in the early years. Finally in additional sensitivity analysis, we used the propensity scores to adjust for potential differences associated with the exposure of primary interest, i.e., between the characteristics of patients who received different treatments during the first year after the cohort entry. To this end, we first used logistic regression to estimate separate propensity scores (PS) for the first-year treatment with 1) MTX and 2) other DMARDs. Both PS estimated the probability of receiving the respective treatment as a function of all time-fixed characteristics available at the entry into the cohort. In two separate analyses, we then included both PS in the multivariable Cox models that also included the cumulative first-year exposures to 1) MTX, 2) other DMARDs, and 3) their interaction, and all time-dependent potential confounders. The difference between the two PS-adjusted models was that the first also included all time-fixed variables, while the second excluded these time-fixed variables.

\section{Results}

We studied 11,365 incident RA patients who were followed for at least 1 year. Baseline characteristics of the RA patients are shown in Table 1 . A majority (67.4\%) were women, with a mean age of 65.3 (standard deviation 14.5) years at cohort entry.

A total of 608 joint replacements occurred during 55,780 person-years (median 4.6 years) of follow up, yielding a rate of 1.09 joint replacements per 100 person-years. The knee $(41.6 \%)$ and hip $(28.2 \%)$ were the most frequent locations for joint replacement. During follow up, $10 \%$ of the patients filled at least one prescription for anti-TNF (ever-users), $58 \%$ for MTX and $65 \%$ had used another DMARD.

Among the alternative measures of drug exposure, the best-fitting multivariable Cox model relied on the cumulative duration of drug use in the first year after cohort entry (Table 2). Joint replacement throughout follow up was significantly lower for patients with higher cumulative 
Table 1 Baseline characteristics of the incident rheumatoid arthritis cohort

\begin{tabular}{|c|c|c|}
\hline Variable & Number & Percent or SD \\
\hline Female & 7,639 & 67.4 \\
\hline Age in years at cohort entry & 65.3 & 14.5 \\
\hline Recipient of social assistance at cohort entry ${ }^{\mathrm{a}}$ & 939 & 8.3 \\
\hline Urban residence ${ }^{a}$ & 8,884 & 78.4 \\
\hline High healthcare use at baseline & 2981 & 26.3 \\
\hline Prior joint replacement & 352 & 3.14 \\
\hline \multicolumn{3}{|l|}{ Comorbidities } \\
\hline Myocardial infarction & 291 & 2.6 \\
\hline Diabetes & 1,668 & 14.7 \\
\hline Osteoporosis & 1,722 & 15.2 \\
\hline Cerebrovascular disease & 54 & 0.48 \\
\hline Acute renal failure & 314 & 2.8 \\
\hline Chronic renal failure & 298 & 2.6 \\
\hline Coronary artery disease & 1,952 & 17.2 \\
\hline COPD-asthma & 2,587 & 22.8 \\
\hline Any type of cancer & 1,326 & 11.7 \\
\hline Osteoarthritis & 3,469 & 30.6 \\
\hline Charlson comorbidity & 1.2 & 1.9 \\
\hline \multicolumn{3}{|l|}{ Drug use prior to cohort entry } \\
\hline GC & 4,360 & 38.5 \\
\hline COXIBs & 4,824 & 42.6 \\
\hline NSAIDs & 4,491 & 39.6 \\
\hline
\end{tabular}

COPD chronic obstructive pulmonary disease, GC glucocorticoids, COXIBS cyclooxygenase-2 inhibitors, NSAIDs nonselective nonsteroidal anti-inflammatory drugs

${ }^{\mathrm{a} D e f i n e d ~ b y ~ c e n s u s ~ t r a c t ~ d a t a ~}$

duration of either MTX ( $\mathrm{HR}=0.95$ per month of use, $95 \%$ CI $0.93,0.97)$ or of other DMARDs (HR $=0.97$ per month of use, $95 \%$ CI $0.95,0.99$ ) within the first year after cohort entry. These results can respectively be translated into a $5 \%$ decrease in the hazard of surgery associated with every additional month of early MTX use in the first year of follow up, and a $3 \%$ decrease in the hazard of surgery for every additional cumulative month of early use of other DMARDs. These associations became much stronger with prolonged use of the relevant drugs in the first year. For example, the early use of MTX or other DMARDs for 6 months was associated with $31 \%(\mathrm{HR}=0.69,95 \% \mathrm{CI}$ $0.59,0.80)$ and $27 \%(\mathrm{HR}=0.8395 \% \mathrm{CI} 0.73,0.95)$ reduction in the hazard of joint replacement surgery during follow up. To facilitate assessment of the impact of early treatment with MTX and/or other DMARDs, Fig. 1 compares the Kaplan-Meier-like curves, for the proportions of patients who remain free of joint replacement in four subgroups, defined based on treatment(s) received during the first year after the cohort entry: 1) users of MTX only (Metho_only); 2) users of other DMARDs only
Table 2 Best-fitting Cox regression model of time to joint replacement surgery

\begin{tabular}{|c|c|c|}
\hline Variable & Hazard ratio & $95 \% \mathrm{Cl}$ \\
\hline Age (at cohort entry) & 1.19 & $1.10,1.28$ \\
\hline Age (at cohort entry) squared & 1.00 & $1.00,1.00$ \\
\hline Female sex & 0.80 & $0.66,0.96$ \\
\hline Recipient of social assistance at cohort entry & 0.89 & $0.59,1.35$ \\
\hline Baseline urban residence & 1.03 & $0.82,1.28$ \\
\hline Baseline regional employment rate ${ }^{a}$ & 1.00 & $0.98,1.01$ \\
\hline Baseline regional family income ${ }^{a}$ & 1.00 & $0.99,1.01$ \\
\hline Baseline regional percent university graduates ${ }^{a}$ & 1.00 & $0.99,1.01$ \\
\hline Extra-articular RA manifestations (time-dependent) & 1.08 & $0.91,1.28$ \\
\hline Rheumatology visits at baseline (log-transformed) & 1.24 & $1.11,1.38$ \\
\hline $\begin{array}{l}\text { Rheumatology visits in follow up, } \\
\text { time dependent (log-transformed) }\end{array}$ & 0.98 & $0.84,1.15$ \\
\hline High health care use at baseline & 1.11 & $0.91,1.35$ \\
\hline Prior joint replacement & 2.55 & $1.90,3.43$ \\
\hline \multicolumn{3}{|l|}{ Baseline comorbidities } \\
\hline Myocardial infarction & 0.86 & $0.48,1.56$ \\
\hline Diabetes & 0.99 & $0.76,1.28$ \\
\hline Osteoporosis & 0.79 & $0.63,0.99$ \\
\hline Cerebrovascular disease & 1.27 & $0.40,4.02$ \\
\hline Acute renal failure & 1.16 & $0.68,1.97$ \\
\hline Chronic renal failure & 1.60 & $0.90,2.84$ \\
\hline Coronary artery disease & 1.01 & $0.81,1.27$ \\
\hline COPD-asthma & 0.96 & $0.77,1.18$ \\
\hline Any type of cancer & 1.01 & $0.72,1.41$ \\
\hline Osteoarthritis & 2.15 & $1.81,2.55$ \\
\hline Charlson Comorbidity & 0.92 & $0.85,1.00$ \\
\hline \multicolumn{3}{|l|}{ Drug use prior to cohort entry } \\
\hline Anti-TNF & 1.00 & $0.35,2.85$ \\
\hline MTX & 1.27 & $0.94,1.70$ \\
\hline Other DMARDs & 1.13 & $0.88,1.45$ \\
\hline Systemic corticosteroids & 1.15 & $0.97,1.38$ \\
\hline COXIBs & 0.97 & $0.82,1.15$ \\
\hline NSAIDs & 0.87 & $0.73,1.03$ \\
\hline
\end{tabular}

Time-dependent cumulative drugs in follow up (years)

$\begin{array}{lll}\text { Anti-TNF } & 1.13 & 0.99,1.29 \\ \text { Other biologic drugs } & 1.37 & 0.61,3.08 \\ \text { Systemic corticosteroids } & 1.01 & 0.94,1.08 \\ \text { COXIBs } & 1.20 & 1.11,1.30 \\ \text { NSAIDs } & 1.31 & 1.20,1.43 \\ \text { Cumulative MTX in first year (months) } & 0.94 & 0.92,0.96 \\ \text { Cumulative other DMARDs in first year months) } & 0.97 & 0.95,0.99\end{array}$

$R A$ rheumatoid arthritis, MTX methotrexate, DMARD disease-modifying anti-rheumatic drug, COPD chronic obstructive pulmonary disease, TNF tumor necrosis factor, COXIB cyclooxygenase-2 inhibitor, NSAID nonsteroidal anti-inflammatory drug

${ }^{a}$ Defined by census tract data 


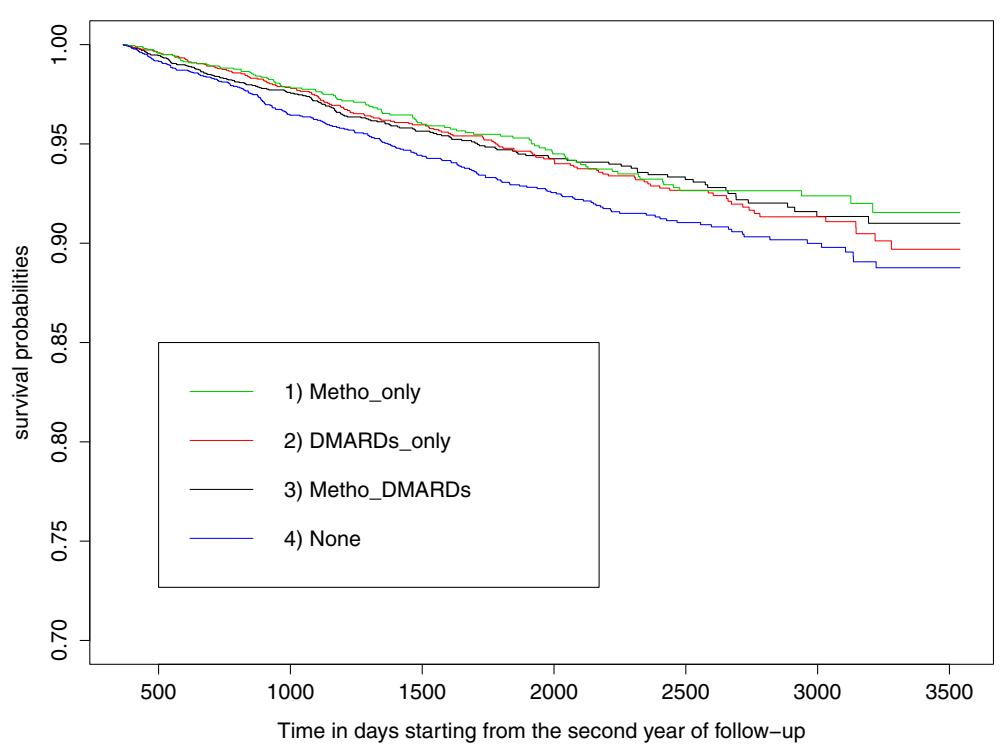

Fig. 1 Kaplan-Meier estimates of time to joint replacement surgery. Groups of drug exposure were based on treatment(s) received during the first year after the cohort entry: 1) users of methotrexate (MTX) only (Metho_only); 2) users of other disease-modifying anti-rheumatic drugs (DMARD) only (DMARDs_only); 3) users of both MTX and DMARDs (Metho_DMARDs); and 4) patients not prescribed either MTX or any other DMARD during the first year of follow up (None)

(DMARDs_only); 3) users of both MTX and DMARDs (Metho_DMARDs); and 4) patients not prescribed either MTX or any other DMARDs during the first year of follow up (None). It is evident that patients treated with MTX and/or other DMARDs in the first year have lower long-term risks of requiring joint replacement, with cumulative risk during the first $4-8$ years of follow up lower by about $2 \%$ (Fig. 1). For example, after about 6 years (2,000 days) the cumulative risk is about $5 \%$ in those treated versus about $7 \%$ in those not treated in the first year.

The model included an interaction between MTX and other DMARDs: patients who used drugs in both classes during the first year after cohort entry had a significantly shorter time to joint replacement than those who used only one of the two drug classes. We were unable to estimate precisely the effect of cumulative duration of anti-TNF agents during follow up (adjusted $\mathrm{HR}=1.13$ per year of use, $95 \% \mathrm{CI} 0.99,1.29)$ due to the small number of anti-TNF users. Cumulative duration of NSAIDs and COXIBs were associated with higher risk of joint replacement (NSAIDs: $\mathrm{HR}=1.31$ per year of use, $95 \%$ CI 1.20 , 1.43; COXIBs: $\mathrm{HR}=1.20$ per year of use, $95 \%$ CI 1.11 , 1.30 ), as were a previous joint replacement (adjusted $\mathrm{HR}=2.55,95 \% \mathrm{CI} 1.90,3.43)$, rheumatology visit at baseline $(\mathrm{HR}=1.24,95 \% \mathrm{CI} 1.11,1.38)$ and diagnosis of OA in the 3 years prior to RA onset (adjusted $\mathrm{HR}=2.15,95 \%$ CI 1.81, 2.55). The sensitivity analyses based on the novel, flexible weighted cumulative exposure model did not improve our estimates of the long-term effects of RA drug exposure. In sensitivity analysis, when we excluded individuals with diagnosis of OA at baseline, the estimates of the cumulative duration of MTX remained similar to our main analysis $(\mathrm{HR}=0.92$ per month of use, $95 \%$ CI 0.89, 0.96). The effect of MTX was also essentially unchanged by exclusion of the patients with hip replacements ( $\mathrm{HR}=0.95,95 \%$ CI 0.91, 0.99). However, in both sensitivity analyses, the confidence interval was widened for the effect of other DMARDs. In the PS-adjusted models, the estimated effects of both main exposures, and of their interaction, were very similar to the estimates from our multivariable model (see Additional file 1).

\section{Discussion}

In our sample of patients with new-onset RA, increased duration of exposure to either MTX or other DMARDs early after the cohort entry was associated with significantly longer time to joint replacement. This finding is consistent with other studies suggesting that early use of DMARDs results in both shortterm and long-term benefits [13-16]. In a populationbased cohort study, patients treated early with DMARDs (within the first year of symptom onset) had a significantly lower progression rate in RA 
compared with patients who initiated treatment 1-5 years after symptom onset [15]. Results from the Utrecht Rheumatoid Arthritis Cohort showed that treatment with conventional DMARDs immediately after diagnosis resulted in less joint surgery when compared with a delayed start [16]. The rationale for a prompt initiation of DMARDs in patients with RA is based on the idea that there is a critical therapeutic window of opportunity, in which disease modification is more successful, possibly because the pathogenetic mechanisms are still in early development [3]. Although biologic therapies were introduced to the Quebec formulary in 2002, our study was unable to make any definitive conclusions about a potential association between these therapies and later need for joint surgery. In the current dataset, very early use (within the first year after RA onset) of biologics was rare.

A caveat to our main findings is that among the cohort members with incident RA, about a quarter did not receive any DMARD therapy during the follow-up time (median of 4.5 years). These individuals tended to be older, and to have more comorbidities at baseline. This combination of increased age and comorbidity may represent a relative contraindication to DMARD use in this subgroup. Conversely, they may represent a subgroup with milder disease. We lack the clinical data to make this determination. This group also had a higher frequency of pre-existing OA and joint replacement surgery even prior to the cohort entry, and therefore would be expected to be at high risk of requiring future joint surgery. This subset of individuals not receiving MTX or other DMARDs, being at high risk of requiring future joint surgery for OA, could explain part of the observed potentially protective effect of cumulative MTX and other DMARD use in the first year of cohort entry. Although the primary indication for joint surgery could not be confirmed from the data, we did seek to address this issue by adjusting for baseline presence of $\mathrm{OA}$ and for current and past exposure to NSAIDs and COXIBs.

In our study, patients who were concurrently or sequentially prescribed both MTX and other DMARDs within the first year after cohort entry had a shorter time to joint replacement, i.e., a higher risk of joint replacement, than users of only one of these drug classes. This perhaps reflects unmeasured confounding by indication, if the use of combination DMARDs is taken as a marker for patients with the most active or severe disease, and accordingly those who are the most susceptible to need early surgery. Confounding by indication has been demonstrated in other observational RA cohorts, with appropriate adjustments made during analysis [17, 18]. While administrative data have the advantage of being population-based and including large numbers of patients, such data do not include the clinical parameters needed to adjust for disease severity/potential confounding by indication.

Given the observational nature of our study, our results are also subject to other possible unmeasured confounders and selection bias. For example, it is possible that receiving either MTX alone or other traditional DMARDs is a marker of unmeasured patient characteristics that would influence willingness to receive joint replacement surgery. Other factors that may be effect modifiers, such as smoking or body mass index, were unavailable in our dataset, although it is unclear how important such variables might be for the current analyses.

Our analyses complement the existing literature. A few studies have investigated whether patients with new-onset RA during the pre-biologic era were more likely to need joint surgery later on, compared to patients with new-onset RA in more recent times (biologics having being available in most developed nations for over a decade) $[19,20]$. One, based on chart review in adults with new-onset RA in the US showed that for the period 1980-1994, $12.1 \%$ of patients had undergone joint surgery 10 years after RA onset. In contrast, the authors found that in a similar, more recent retrospective cohort of new-onset RA, followed from 1995-2007, only $6.0 \%$ had had joint surgery after 10 years. The authors interpreted the lower joint replacement rate as indicative of improved RA treatments [19]. Similar conclusions were reached in a Finnish study [21]. Conversely, a multicenter study in Japan did not observe a decrease in the rates of RA-associated orthopedic surgery from 1998-2008, despite a steady increase in the proportion of patients using biologics from 2004 (1.8\%) to 2007 (10.0\%) [22]. One possible explanation for that finding is that in more recent years, patients who seek greater levels of activity may be undergoing joint surgery earlier than patients in the past. In addition, the study from Japan included not just joint replacements but also other types of surgery. Finally, technological advancements mean that patients today have more joint-preserving surgical options than in the past.

The strengths of our study are its population-based nature and the availability of detailed drug prescription data. However, data from administrative sources lack primary clinical information on important clinical characteristics, which makes it difficult to adjust for RA severity. Similar to other database studies of drugs use 
and their effects in RA [23, 24], we did use proxies of RA severity, including extra-articular manifestations of RA, number of rheumatology visits, and use of concomitant medications like glucocorticoids. This approach has been used by other authors and appears to control to some extent for disease severity [25], though it is not ideal.

Time until joint replacement, as measured in our study, is influenced not only by necessity, but also by healthcare access. For example, individuals with higher socioeconomic status tend to have better access to care, and thus, have shorter waiting times even within publicly funded healthcare systems, to receive elective surgery [26]. In our study, we adjusted for variables related to socioeconomic status, such as being on social assistance, and ecological measures such as family income, employment, and education [27]. We were not able to address other issues related to accessibility, such as wait times to either consult a surgeon or obtain surgery or difficulties on the part of patients to attend appointments. Finally, we have to take some aspects into account when extrapolating our results. The rate of biologic use represents an average over time and accounts for periods when these drugs were not yet widely available. Nevertheless, the frequency of use of both biologics and non-biologic DMARDs found in our study is not greatly different from those documented in similar studies using administrative databases [28]. Moreover, the access to anti-TNF agents in Canada is comparable to that observed in countries with a similar income, with the exception of the USA [29]. All things considered, we believe the results of this study can be informative for other countries with similar sociodemographics characteristics and healthcare access.

\section{Conclusions}

In summary, our study suggests that higher cumulative use of MTX and higher cumulative use of nonMTX traditional DMARDs within the first year after the cohort entry may be associated with longer time to joint surgery. A causal link cannot be firmly established with these observational data; however, these real-world findings may be consistent with jointsparing effects suggested by other data. Given that biologic therapies were only recently introduced to the Quebec formulary, our study was unable to make any definitive conclusions about the potential effect of these novel therapies and later need for surgery, but analyses of future data using similar methods should investigate this issue.

\section{Appendix 1}

\author{
List of CCI/CCP procedure codes for joint replacement \\ Canadian Classification of Health Interventions \\ Code Description \\ 1EL53 Implantation of internal device, temporomandibular joint [TMJ] \\ 1SQ53 Implantation of device, pelvis \\ 1TA53 Implantation of internal device, shoulder joint \\ 1TM53 Implantation of internal device, elbow joint \\ 1UB53 Implantation of internal device, wrist joint \\ 1UC53 Implantation of internal device, distal radioulnar joint and carpal \\ joints and bones \\ 1UG53 Implantation of internal device, metacarpophalangeal joint(s) \\ 1UH53 Implantation of internal device, first metacarpophalangeal joint \\ 1UK53 Implantation of internal device, interphalangeal joints of hand \\ 1UM53 Implantation of internal device, first interphalangeal joint \\ of hand \\ 1VA53 Implantation of internal device, hip joint \\ 1VG53 Implantation of internal device, knee joint \\ 1VP53 Implantation of internal device, patella \\ 1WA53 Implantation of internal device, ankle joint \\ 1WE53 Implantation of internal device, tarsal bones and intertarsal \\ joints [hindfoot, midfoot]
}

1W153 Implantation of internal device, first metatarsal bone and first metatarsophalangeal joint

1WJ53 Implantation of internal device, tarsometatarsal joints, metatarsal bones and metatatarsophalangeal joints [forefoot]

1WM53 Implantation of internal device, interphalangeal joints of toe

1WN53 Implantation of internal device, first interphalangeal joint of toe

Canadian Classification of Diagnostic, Therapeutic and Surgical Procedures (CCP)

\begin{tabular}{ll} 
Code & Description \\
9341 & Total knee replacement (geomedic) (polyc \\
9348 & Total ankle replacement \\
9350 & Total hip replacement \\
9351 & Total hip replacement with methyl methacrylate \\
9359 & Other total hip replacement \\
9360 & Other arthroplasty of hip \\
9361 & Replacement of head of femur with use of methyl methacrylate \\
9362 & Other replacement of head of femur \\
9363 & Replacement of acetabulum with use of methyl methacrylate \\
9364 & Other replacement of acetabulum \\
9370 & Arthroplasty of hand and finger \\
9371 & Arthroplasty of hand and finger with synthetic prosthesis \\
9380 & Arthroplasty of upper extremity, except hand \\
9381 & Arthroplasty of shoulder with synthetic \\
9384 & Arthroplasty of elbow with synthetic prosthesis \\
9386 & Arthroplasty of carpals with synthetic prosthesis \\
\hline
\end{tabular}




\section{Additional file}

Additional file 1: Propensity score analysis. Results of the propensity score analysis for the main exposure drug groups and comparison with the best-fitting multivariable Cox model. (DOC $39 \mathrm{~kb}$ )

\section{Abbreviations}

AIC: Akaike Information Criterion; anti-TNF: anti-tumor necrosis factor; CCl: Canadian Classification of Health Intervention; CCP: Canadian Classification of Diagnostic, Therapeutic and Surgical Procedures; COPD: chronic obstructive pulmonary disease; COXIBs: cyclooxygenase-2 inhibitors; DMARDs: disease-modifying anti-rheumatic drugs; HR: hazard ratio; ICD: International Classification of Diseases; MTX: methotrexate; NSAIDs: nonselective nonsteroidal anti-inflammatory drugs; OA: osteoarthritis; PH: proportional hazards; PS: propensity scores; RA: rheumatoid arthritis; RAMQ: Quebec Health Insurance Program.

\section{Competing interests}

This study was funded by the Canadian Institutes of Health Research, through the Drug Safety and Effectiveness Network. Dr. Paul R. Fortin presently holds a tier 1 Canada Research Chair on Systemic Autoimmune Rheumatic Diseases at Université Laval. Dr. Bombardier holds a Canada Research Chair in Knowledge Transfer for Musculoskeletal Care (2002-2016) and a Pfizer Research Chair in Rheumatology. All other coauthors have nothing to disclose

\section{Authors' contributions}

All authors have made substantial contributions to conception and design and/or analysis or interpretation of data. CSM, MA, DL, GB, PRF, LB, CBo, JW, $J G H, D F, W M, C P, C B a, S E$, and SB contributed to the study design, data analysis, interpretation and preparation of results. MEB and YW contributed to data preparation, data analysis and interpretation and presentation of results. All authors contributed to the manuscript revision process and addressed important intellectual content. All authors read and approved the final manuscript for publication.

\section{Acknowledgments}

The CAnadian Network for Advanced Interdisciplinary Methods for comparative effectiveness research (CAN-AIM) group is supported by the Canadian Institutes of Health Research, through the Drug Safety and Effectiveness Network. Disclosures: Dr. Paul R. Fortin presently holds a tier 1 Canada Research Chair on Systemic Autoimmune Rheumatic Diseases at Université Laval. Dr. Bombardier holds a Canada Research Chair in Knowledge Transfer for Musculoskeletal Care (2002-2016) and a Pfizer Research Chair in Rheumatology.

\section{Author details}

${ }^{1}$ Department of Epidemiology, Biostatistics and Occupational Health, McGill University, Montreal, QC, Canada. ${ }^{2}$ Division of Clinical Epidemiology, McGill University Health Centre, 687 Pine Avenue West, V-Building (V2.09), Montreal, QC H3A 1A1, Canada. ${ }^{3}$ Department of Medicine, University of British Columbia, Vancouver, BC, Canada. ${ }^{4}$ Department of Medicine, Université de Sherbrooke, Sherbrooke, QC, Canada. ${ }^{5}$ Division of Rheumatology, Department of Medicine, Université Laval, Quebec City, QC, Canada. ${ }^{6}$ Division of Rheumatology, University of Toronto, Toronto, ON, Canada. ${ }^{7}$ Institute for Clinical Evaluative Sciences, Toronto, ON, Canada. ${ }^{8}$ Division of Rheumatology, Department of Medicine, Dalhousie University, Halifax, NS, Canada. ${ }^{9}$ École de Réadaptation, Université de Montréal, Montreal, QC, Canada. ${ }^{10}$ Department of Medicine, University of Alberta, Edmonton, AB, Canada. ${ }^{11}$ Department of Internal Medicine, University of Manitoba, Winnipeg, MB, Canada. ${ }^{12}$ Department of Medicine, University of Calgary, Calgary, AB, Canada. ${ }^{13}$ Department of Community Health Sciences, University of Calgary, Calgary, $A B$, Canada.

Received: 7 May 2015 Accepted: 10 July 2015

Published online: 03 August 2015

\section{References}

1. Widdifield J, Paterson JM, Bernatsky S, Tu K, Tomlinson G, Kuriya B, et al. The epidemiology of rheumatoid arthritis (RA) in Ontario. Canada Arthritis Rheum. 2013;66:786-93.

2. Emery P, Breedveld FC, Hall S, Durez P, Chang DJ, Robertson D, et al. Comparison of methotrexate monotherapy with a combination of methotrexate and etanercept in active, early, moderate to severe rheumatoid arthritis (COMET): a randomised, double-blind, parallel treatment trial. Lancet. 2008;372:375-82.

3. van Nies JA, Krabben A, Schoones JW, Huizinga TW, Kloppenburg M, van der Helm-van Mil AH. What is the evidence for the presence of a therapeutic window of opportunity in rheumatoid arthritis? A systematic literature review. Ann Rheum Dis. 2013;73:861-70.

4. Boers M. Understanding the window of opportunity concept in early rheumatoid arthritis. Arthritis Rheum. 2003;48:1771-4.

5. James D, Young A, Kulinskaya E, Knight E, Thompson W, Ollier W, et al. Orthopaedic intervention in early rheumatoid arthritis. Occurrence and predictive factors in an inception cohort of 1064 patients followed for 5 years. Rheumatology (Oxford). 2004;43:369-76.

6. Jamsen E, Virta LJ, Hakala M, Kauppi MJ, Malmivaara A, Lehto MU. The decline in joint replacement surgery in rheumatoid arthritis is associated with a concomitant increase in the intensity of anti-rheumatic therapy: a nationwide register-based study from 1995 through 2010. Acta Orthop. 2013;84:331-7.

7. Widdifield J, Bernatsky S, Paterson JM, Tu K, Ng R, Thorne JC, et al. Accuracy of Canadian health administrative databases in identifying patients with rheumatoid arthritis: a validation study using the medical records of rheumatologists. Arthritis Care Res (Hoboken). 2013;65:1582-91.

8. Abrahamowicz M, Tamblyn R. Drug utilization patterns. In: Armitage P, Colton T, editors. Encyclopedia of Biostatistics. 2nd ed. Chichester: John Wiley \& Sons; 2005. p. 1533-53.

9. Abrahamowicz M, Bartlett G, Tamblyn R, du Berger R. Modeling cumulative dose and exposure duration provided insights regarding the associations between benzodiazepines and injuries. J Clin Epidemiol. 2006;59:393-403.

10. Abrahamowicz M, Beauchamp ME, Sylvestre MP. Comparison of alternative models for linking drug exposure with adverse effects. Stat Med. 2012;31:1014-30

11. Akaike H. A new look at the statistical model identification. IEEE Trans Autom Control. 1973;19:716-23.

12. Sylvestre MP, Abrahamowicz M. Flexible modeling of the cumulative effects of time-dependent exposures on the hazard. Stat Med. 2009;28:3437-53.

13. Finckh $\mathrm{A}$, Liang $\mathrm{MH}$, van Herckenrode $\mathrm{CM}$, de Pablo P. Long-term impact of early treatment on radiographic progression in rheumatoid arthritis: $A$ meta-analysis. Arthritis Rheum. 2006;55:864-72.

14. Korpela M, Laasonen L, Hannonen P, Kautiainen H, Leirisalo-Repo M, Hakala M, et al. Retardation of joint damage in patients with early rheumatoid arthritis by initial aggressive treatment with disease-modifying antirheumatic drugs: five-year experience from the FIN-RACo study. Arthritis Rheum. 2004;50:2072-81.

15. Kyburz D, Gabay C, Michel BA, Finckh A. The long-term impact of early treatment of rheumatoid arthritis on radiographic progression: a population-based cohort study. Rheumatology (Oxford). 2011;50:1106-10.

16. Verstappen SM, Hoes JN, Ter Borg EJ, Bijlsma JW, Blaauw AA, van Albada-Kuipers GA, et al. Joint surgery in the Utrecht Rheumatoid Arthritis Cohort: the effect of treatment strategy. Ann Rheum Dis. 2006;65:1506-11.

17. Bukhari MA, Wiles NJ, Lunt M, Harrison BJ, Scott DG, Symmons DP, et al. Influence of disease-modifying therapy on radiographic outcome in inflammatory polyarthritis at five years: results from a large observational inception study. Arthritis Rheum. 2003;48:46-53.

18. Wiles NJ, Lunt M, Barrett EM, Bukhari M, Silman AJ, Symmons DP, et al. Reduced disability at five years with early treatment of inflammatory polyarthritis: results from a large observational cohort, using propensity models to adjust for disease severity. Arthritis Rheum. 2001;44:1033-42.

19. Louie GH, Ward MM. Changes in the rates of joint surgery among patients with rheumatoid arthritis in California, 1983-2007. Ann Rheum Dis. 2007;69:868-71.

20. Weiss RJ, Ehlin A, Montgomery SM, Wick MC, Stark A, Wretenberg P. Decrease of RA-related orthopaedic surgery of the upper limbs between 1998 and 2004: data from 54,579 Swedish RA inpatients. Rheumatology (Oxford). 2008;47:491-4. 
21. Sokka T, Kautiainen $H$, Hannonen P. Stable occurrence of knee and hip total joint replacement in Central Finland between 1986 and 2003: an indication of improved long-term outcomes of rheumatoid arthritis. Ann Rheum Dis. 2007;66:341-4

22. Momohara S, Tanaka S, Nakamura H, Mibe J, Iwamoto T, Ikari K, et al. Recent trends in orthopedic surgery performed in Japan for rheumatoid arthritis. Mod Rheumatol. 2011;21:337-42.

23. Ting G, Schneeweiss S, Scranton R, Katz JN, Weinblatt ME, Young M, et al. Development of a health care utilisation data-based index for rheumatoid arthritis severity: a preliminary study. Arthritis Res Ther. 2008;10:R95.

24. Wolfe F, Michaud K, Simon T. Can severity be predicted by treatment variables in rheumatoid arthritis administrative data bases? J Rheumatol. 2006:33:1952-6.

25. Dixon WG, Hyrich KL, Watson KD, Lunt M, Symmons DP. Influence of anti-TNF therapy on mortality in patients with rheumatoid arthritis-associated interstitial lung disease: results from the British Society for Rheumatology Biologics Register. Ann Rheum Dis. 2010;69:1086-91.

26. Laudicella M, Siciliani L, Cookson R. Waiting times and socioeconomic status: evidence from England. Soc Sci Med. 2010;74:1331-41.

27. Hawker GA, Wright JG, Glazier RH, Coyte PC, Harvey B, Williams JI, et al. The effect of education and income on need and willingness to undergo total joint arthroplasty. Arthritis Rheum. 2002:46:3331-9.

28. Schmajuk G, Solomon DH, Yazdany J. Patterns of disease-modifying antirheumatic drug use in rheumatoid arthritis patients after 2002: a systematic review. Arthritis Care Res (Hoboken). 2013;65:1927-35.

29. Jonsson B, Kobelt G, Smolen J. The burden of rheumatoid arthritis and access to treatment: uptake of new therapies. Eur J Health Econ. 2008:8:561-86.

\section{Submit your next manuscript to BioMed Central and take full advantage of:}

- Convenient online submission

- Thorough peer review

- No space constraints or color figure charges

- Immediate publication on acceptance

- Inclusion in PubMed, CAS, Scopus and Google Scholar

- Research which is freely available for redistribution 